\title{
Emergency ureteroscopic treatment for upper urinary tract calculi obstruction associated with acute renal failure: feasible or not?
}

Yang S, Qian H, Song C, Xia Y, Cheng F, Zhang C

Division of Urology, Renming Hospital of Wuhan University, Wuhan City, Hubei Province, People's Republic of China

J Endourol. 2010; 24: 1721-4

Purpose: To determine the efficacy and safety of emergency ureteroscopy (URS) and holmium:yttrium-aluminum-garnet (Ho:YAG) laser lithotripsy for ureteral calculi that are associated with acute renal failure (ARF).

Patients and Methods: We retrospectively evaluated a cohort of 49 patients who underwent URS from November 2005 to November 2008 for ARF that was caused by calculi obstruction of the upper urinary tract. The mean (maximal diameter) stone size was $1.48 \mathrm{~cm}$. Acute renal failure was demonstrated by oliguria or anuria and marked increase in serum creatinine and blood urea nitrogen levels. All the patients were treated with URS and Ho:YAG laser lithotripsy emergently. Ureteral stent placement was performed in all cases after lithotripsy. A plain film of the kidneys, ureters, and bladder and abdominal ultrasonography were performed to evaluate efficacy of treatment on the first day postoperatively. Serum creatinine and blood urea nitrogen levels and urine volume were successively monitored until they returned to normal. All patients had postoperative imaging, including ultrasonography and excretory urography, to confirm stone clearance and exclude late obstructive complications 3 months after URS.

Results: URS and laser lithotripsy were successfully performed in all patients. There were no major intraoperative complications, and no procedure was converted to open surgery. The mean operative time was 35 minutes. The successful fragmentation rate was $95.5 \%$. The overall stone-free rate was $91.8 \%$. Normal renal function returned in 46 (93.8\%) patients within 7 days. No postoperative ureteral stricture occurred after 3 months.

Conclusions: URS and Ho:YAG laser lithotripsy represent an effective and safe modality for treating patients with ARF that is caused by calculi obstruction of the upper urinary tract in strictly selected situations.

\section{Editorial Comment}

The authors report remarkable results ( $92 \%$ stone-free rates, ability to reach all proximal ureteral stones) despite using a relatively large semi-rigid ureteroscope and not utilizing a flexible ureteroscope for any cases. Indeed, they report treating stones larger than $3 \mathrm{~cm}$ in size in less than 1 hour. They utilized higher energy settings $(1.8 \mathrm{~J})$, which may facilitate more rapid fragmentation, but have been reported in "in vitro" studies to lead to larger stone fragments. It may be that the longer stenting times (4-12 weeks) helped facilitate stone passage without the development of recurrent obstruction. The authors provide important information concerning the safety of ureteroscopy in the face of acute renal failure, and the course of resolution of the renal failure with alleviation of the obstruction. Likely, the short operative time is critical in these patients to minimize the risks of peri-operative complication related to irrigant fluid absorption and length of anesthetic.

Dr. Manoj Monga

Director, Stevan B. Streem Center for Endourology \& Stone Disease Glickman Urological \& Kidney Institute

The Cleveland Clinic

Cleveland, Ohio, USA

E-mail: endourol@yahoo.com 